\title{
SOLVABILITY OF SYSTEMS OF LINEAR OPERATOR EQUATIONS
}

\author{
RONG-QING JIA, SHERMAN RIEMENSCHNEIDER, AND ZUOWEI SHEN
}

(Communicated by J. Marshall Ash)

\begin{abstract}
Let $G$ be a semigroup of commuting linear operators on a linear space $S$ with the group operation of composition. The solvability of the system of equations $l_{i} f=\phi_{i}, i=1, \ldots, r$, where $l_{i} \in G$ and $\phi_{i} \in S$, was considered by Dahmen and Micchelli in their studies of the dimension of the kernel space of certain linear operators. The compatibility conditions $l_{j} \phi_{i}=l_{i} \phi_{j}$, $i \neq j$, are necessary for the system to have a solution in $S$. However, in general, they do not provide sufficient conditions. We discuss what kinds of conditions on operators will make the compatibility sufficient for such systems to be solvable in $S$.
\end{abstract}

\section{INTRODUCTION}

This paper deals with the solvability of systems of linear operator equations. In particular, we will provide necessary and sufficient conditions under which certain kinds of systems of differential and difference equations are solvable.

Let $G$ be a semigroup of commuting linear operators on a linear space $S$ with the group operation of composition. The solvability of the system of equations

$$
l_{i} f=\phi_{i}, \quad i=1, \ldots, r,
$$

where $l_{i} \in G$ and $\phi_{i} \in S$, was considered by Dahmen and Micchelli in their studies of exponential box splines $\left(\right.$ see $\left.\left[D_{1}\right]\right)$. It is clear that the compatibility conditions

$$
l_{j} \phi_{i}=l_{i} \phi_{j} \quad(i \neq j)
$$

are necessary for the system (1.1) to have a solution in $S$. However, in general, they do not provide sufficient conditions for the system (1.1) to be solvable in $S$. In $\S 2$, we shall discuss what kind of conditions on operators will make the conditions (1.2) sufficient for the system (1.1) to be solvable in $S$.

Received by the editors November 8, 1991 and, in revised form, June 23, 1992.

1991 Mathematics Subject Classification. Primary 41A15, 41A63, 13A15, 35G05, 39A70.

Key words and phrases. Systems of operator equations, multivariate approximation, polynomial ideals, linear partial differential equations, linear partial difference equations.

Research supported in part by NSERC Canada under Grant \#A7687 and by NATO Collaborative Research under Grant CRG 901018. 
A special interesting case is when $G$ consists of linear partial differential operators induced by polynomials and $S$ is the linear space of formal power series. Let $K$ be an arbitrary field, and $K^{s}$ the $s$-dimensional affine space over $K$. Denote by $\Pi\left(K^{s}\right)$ the ring of polynomials in $s$ indeterminates $X_{1}, \ldots, X_{s}$ over the field $K$. We shall use the standard multi-index notation. Let $\mathbb{N}$ denote the set of nonnegative integers. For a multi-index $\beta=\left(\beta_{1}, \ldots, \beta_{s}\right) \in \mathbb{N}^{s}$ we define

$$
X^{\beta}:=X_{1}^{\beta_{1}} \cdots X_{s}^{\beta_{s}} .
$$

A formal power series in $X_{1}, \ldots, X_{s}$ has the form $\sum_{\beta \in \mathbb{N}^{s}} b_{\beta} X^{\beta}$, where all $b_{\beta} \in K$. For a multi-index $\alpha=\left(\alpha_{1}, \ldots, \alpha_{s}\right) \in \mathbb{N}^{s}$, the formal differential operator $D^{\alpha}$ is defined by

$$
D^{\alpha}\left(\sum_{\beta \in \mathbb{N}^{s}} b_{\beta} X^{\beta}\right)=\sum_{\beta \in \mathbb{N}^{s}} b_{\beta} \frac{\beta !}{(\beta-\alpha) !} X^{\beta-\alpha},
$$

where we have adopted the convention that $X^{\beta-\alpha}=0$, if $\beta_{j}<\alpha_{j}$ for some $j$. Given a polynomial $p \in \Pi\left(K^{s}\right), p(X)=\sum_{\alpha} a_{\alpha} X^{\alpha}$, we denote by $p(D)$ the partial differential operator induced by $p$ :

$$
p(D):=\sum_{\alpha} a_{\alpha} D^{\alpha}
$$

The corresponding system (1.1) becomes a system of partial differential equations:

$$
p_{i}(D) f=\phi_{i}, \quad i=1, \ldots, r .
$$

When $K$ is the complex field $\mathbb{C}$ and $S$ is the linear space of formal power series over $\mathbb{C}$, Ehrenpreis [E, Theorem 6.1] proved that the above system (1.3) has a solution in $S$ if and only if whenever $q_{1}, \ldots, q_{r} \in \Pi\left(\mathbb{C}^{s}\right)$ satisfy

$$
\sum_{i=1}^{r} q_{i} p_{i}=0
$$

it follows that

$$
\sum_{i=1}^{r} q_{i}(D) \phi_{i}=0
$$

He also remarked that it would be of interest to prove an analogous result for the ring of polynomials over an abstract field (see [E, p. 173]).

In $\left[\mathrm{DM}_{2}\right]$, Dahmen and Micchelli investigated the solvability of the system (1.3) when $S$ is the linear space of polynomials over $\mathbb{C}$. In particular, they obtained the following result.

(1.4) Theorem $\left[\mathrm{DM}_{2}\right]$. Let $p_{1}$ and $p_{2}$ be any homogeneous polynomials on $\mathbb{R}^{2}$ with no common nontrivial zeros. Then the system of equations

$$
p_{1}(D) f=\phi_{1}, \quad p_{2}(D) f=\phi_{2}
$$

has a solution if and only if

$$
p_{2}(D) \phi_{1}=p_{1}(D) \phi_{2}
$$


It should be pointed out that this theorem is true only if $p_{1}$ and $p_{2}$ have no common nontrivial zeros in $\mathbb{C}^{2}$ (rather than $\mathbb{R}^{2}$ ). Moreover, it is not necessary that the polynomials $p_{1}$ and $p_{2}$ be homogeneous.

In $\S 3$ we shall investigate the solvability of the system (1.3) when $K$ is an algebraically closed field of characteristic zero and $S$ is the linear space of polynomials (or exponential polynomials) over $K$. We shall show, among other things, that if $p_{1}, \ldots, p_{s} \in \Pi\left(K^{s}\right)$ have only finitely many common zeros in $K^{s}$, then the system of partial differential equations

$$
p_{j}(D) f=\phi_{j}, \quad j=1, \ldots, s,
$$

where $\phi_{j} \in \Pi\left(K^{s}\right)$, has a solution in $\Pi\left(K^{s}\right)$ if and only if

$$
p_{j}(D) \phi_{k}=p_{k}(D) \phi_{j}, \quad j \neq k \text {. }
$$

Furthermore, we shall establish a similar result for difference equations. Our work is of some algebraic flavor.

Our investigation of the system (1.1) of operator equations relies on the concept of $s$-dimensional additivity for a semigroup $G$, which was first introduced in [S]. Let $G$ be a commutative semigroup of linear operators on a linear space $S$. We say that $G$ possesses $s$-dimensional additivity if for any $s+1$ elements $l_{1}, l_{1}^{\prime}, l_{2}, \ldots, l_{s} \in G$,

$\operatorname{dim}\left(\operatorname{ker}\left(l_{1} l_{1}^{\prime}, l_{2}, \ldots, l_{s}\right)\right)=\operatorname{dim}\left(\operatorname{ker}\left(l_{1}, l_{2}, \ldots, l_{s}\right)\right)+\operatorname{dim}\left(\operatorname{ker}\left(l_{1}^{\prime}, l_{2}, \ldots, l_{s}\right)\right)$, where $\operatorname{ker}\left(\tilde{l}_{1}, \ldots, \tilde{l}_{s}\right):=\left\{f \in S: \tilde{l}_{j} f=0, j=1, \ldots, s\right\}$.

For any $\theta \in K^{s}$, we denote by $e_{\theta}$ the formal power series $\sum_{\alpha \in \mathbb{N}^{s}} \theta^{\alpha} X^{\alpha} / \alpha$ !. We call $e_{\theta}$ a pure exponential. A formal power series is called an exponential polynomial if it is a linear combination of products of polynomials with pure exponentials. From the definition of $e_{\theta}$ we see that for a polynomial $p$,

$$
p(D) e_{\theta}=p(\theta) e_{\theta} \text {. }
$$

(1.5) Example. Let $S$ be the linear space of all exponential polynomials on $K^{s}$, where $K$ is an algebraically closed field of characteristic zero. Let $G:=$ $G_{\Pi\left(K^{s}\right)}(D)$ be the collection of all $p(D), p \in \Pi\left(K^{s}\right)$. Then $G$ is a commutative semigroup and possesses $s$-dimensional additivity on $S$ (see [JRS, Corollary 3.5]).

Let $\mathbb{Z}$ be the set of integers and $s$ be a positive integer. A mapping from $\mathbb{Z}^{s}$ to $K$ is called an $s$-variate $K$-sequence. The linear space of all $s$-variate $K$-sequences will be denoted by $A$. We wish to consider shift operators on $A$. If $\alpha \in \mathbb{N}^{s}$ is a multi-index, then we define $\tau^{\alpha}$ to be the shift operator given by

$$
\tau^{\alpha} f:=f(\cdot+\alpha) \text { for all } f \in A \text {. }
$$

For a polynomial $p \in \Pi\left(K^{s}\right), p(X)=\sum_{\alpha} a_{\alpha} X^{\alpha}$, we denote by $p(\tau)$ the difference operator induced by $p$ :

$$
p(\tau):=\sum_{\alpha} a_{\alpha} \tau^{\alpha}
$$

If $g \in \Pi\left(K^{s}\right)$, then the sequence given by $\beta \mapsto g(\beta), \beta \in \mathbb{Z}^{s}$, will be called a polynomial sequence.

For any $\theta=\left(\theta_{1}, \ldots, \theta_{s}\right) \in(K \backslash\{0\})^{s}$, we denote by $\theta^{()}$the sequence

$$
\beta \mapsto \theta^{\beta}:=\theta_{1}^{\beta_{1}} \cdots \theta_{s}^{\beta_{s}}, \quad \beta=\left(\beta_{1}, \ldots, \beta_{s}\right) \in \mathbb{Z}^{s} .
$$


We call $\theta^{()}$a pure exponential sequence. A sequence is called an exponential polynomial sequence if it is a linear combination of products of polynomial sequences with pure exponential sequences. It is easily seen that for a polynomial $p$,

$$
p(\tau)\left(\theta^{()}\right)=p(\theta)\left(\theta^{()}\right) .
$$

(1.6) Example. Let $S$ be the linear space of all exponential polynomial $K$ sequences on $\mathbb{Z}^{s}$. Let $G:=G_{\Pi\left(K^{s}\right)}(\tau)$ be the collection of all $p(\tau), p \in \Pi\left(K^{s}\right)$. Then $G$ is a commutative semigroup and possesses $s$-dimensional additivity on $S$ (see [JRS, Theorem 4.4]).

Why does $s$-dimensional additivity play an important role here? As we pointed out in [RJS], if for $G$ and any system of equations (1.1) with $r=s$, the compatibility conditions are sufficient for solvability, then $G$ has the property of $s$-dimensional additivity. On the other hand, we will show that under some mild conditions on linear partial differential and difference operators, $s$ dimensional additivity implies the solvability of the system of equations (1.1) whenever the compatibility conditions hold.

\section{SOLVABILITY OF LINEAR OPERATOR EQUATIONS}

We discuss in this section possible conditions on $G$ and $l_{j}$, under which the compatibility conditions (1.2) are sufficient to guarantee the solvability of the system (1.1).

Let $M$ be a subspace of $S$. An element $l \in G$ is called nilpotent on $M$ if for any $\phi \in M$, there exists a positive integer $m$ such that $l^{m} \phi=0(m$ may depend on $\phi$ ). We say that $M$ is compatible with $G$ if the following two conditions are satisfied:

(i) $M$ is invariant under $G$, i.e., for any $l \in G, l(M) \subseteq M$; and

(ii) for any $l \in G,\left.l\right|_{M}$ is either invertible or nilpotent.

(2.1) Example. In Example (1.5), let $M=e_{\theta} \Pi\left(K^{s}\right)$, where $\theta \in K^{s}$. Then $M$ is compatible with $G_{\Pi\left(K^{s}\right)}(D)$.

For any polynomials $p$ and $\phi$ on $K^{s}$, we have

$$
p(D)\left(e_{\theta} \phi\right)=e_{\theta} p(\theta+D) \phi .
$$

This shows that $M$ is invariant under $G$. Observe that as a mapping on $\Pi\left(K^{s}\right)$, $p(\theta+D)$ is degree preserving if $p(\theta) \neq 0$; otherwise, it is degree reducing. Thus, if $p(\theta)=0$ and $\phi$ is a polynomial of degree $k$, then it follows from (2.2) that $p^{k+1}(D)\left(e_{\theta} \phi\right)=0$. In other words, $p(\theta)=0$ implies that $p(D)$ is nilpotent on $M$. If $p(\theta) \neq 0$, then multiplying by a suitable constant, we may assume that $p(\theta)=1$. Let $q:=1-p$. Then $q(\theta)=0$. Applying (2.2) again, we see that for a given polynomial $\phi$ of degree $\leq k, p(D) f=e_{\theta} \phi$ if and only if

$$
f=\left(1+q(D)+\cdots+q^{k}(D)\right)\left(e_{\theta} \phi\right)
$$

(since $\left.1-q^{k+1}(D)=p(D)\left(1+\cdots+q^{k}(D)\right)\right)$. Thus $p(\theta) \neq 0$ implies that $p(D)$ is invertible. This verifies (ii).

(2.3) Example. In Example (1.6), let $M=\theta^{()} \Pi\left(K^{s}\right)$, where $\theta \in(K \backslash\{0\})^{s}$. Then $M$ is compatible with $G_{\Pi\left(K^{s}\right)}(\tau)$. 
Let $\Pi_{k}$ denote the set of all polynomials on $K^{s}$ of degree at most $k$. We observe that for $\phi \in \Pi_{k}$,

$$
p(\tau)\left(\theta^{()} \phi\right)-p(\theta)\left(\theta^{()} \phi\right) \in \theta^{()} \Pi_{k-1} .
$$

This shows that $M$ is invariant under $G$. Moreover, $\left.p(\tau)\right|_{M}$ is either nilpotent or invertible depending on whether or not $p(\theta)=0$.

(2.4) Theorem. Let $G$ be a commutative semigroup of linear operators on $S$, and let $l_{1}, \ldots, l_{r}$ be elements of $G$. Assume that one of them, say $l_{1}$, is invertible on $S$. Then for given $\phi_{1}, \ldots, \phi_{r}$ in $S$, the system of equations

$$
l_{j} f=\phi_{j}, \quad j=1, \ldots, r,
$$

has a solution in $S$ if and only if the following compatibility conditions hold:

$$
l_{j} \phi_{k}=l_{k} \phi_{j}, \quad 1 \leq j<k \leq r .
$$

Proof. We claim that $l_{1}^{-1}$ commutes with each $l_{j}, j=1, \ldots, r$. Indeed, it follows from $l_{1} l_{j}=l_{j} l_{1}$ that

$$
l_{1}^{-1} l_{j}=l_{1}^{-1}\left(l_{j} l_{1}\right) l_{1}^{-1}=l_{1}^{-1}\left(l_{1} l_{j}\right) l_{1}^{-1}=l_{j} l_{1}^{-1} .
$$

Let $f=l_{1}^{-1} \phi_{1}$. Then $f$ is a solution to the system.

(2.5) Theorem. Let $G$ be a commutative semigroup of linear operators on $S$ which possesses s-dimensional additivity. Suppose that $S$ is a direct sum of two subspaces $M$ and $N$ which are invariant under $G$. Moreover, assume that $l_{1}, \ldots, l_{s} \in G$ are nilpotent on $M$ and have the property $\operatorname{dim}\left(\operatorname{ker}\left(l_{1}, \ldots, l_{s}\right)\right)<$ $\infty$. Let $r \in\{1, \ldots, s\}$. Then for given $\phi_{1}, \ldots, \phi_{r} \in M$, the system of operator equations

$$
l_{j} f=\phi_{j}, \quad j=1, \ldots, r,
$$

has a solution in $M$ if and only if the following compatibility conditions hold:

$$
l_{j} \phi_{k}=l_{k} \phi_{j}, \quad 1 \leq j<k \leq r .
$$

Proof. Obviously, the compatibility conditions (2.7) are necessary for the system (2.6) to have a solution.

For the sufficiency part, we first consider the special case where $r=s$ and $\phi_{2}=\cdots=\phi_{s}=0$. Since $\left.l_{1}\right|_{M}$ is nilpotent, there exists a positive integer $m$ such that $l_{1}^{m} \phi_{1}=0$. Let

$$
\begin{aligned}
H & :=\operatorname{ker}\left(l_{1}^{m+1}, l_{2}, \ldots, l_{s}\right), \\
H^{\prime} & :=\operatorname{ker}\left(l_{1}^{m}, l_{2}, \ldots, l_{s}\right), \\
H^{\prime \prime} & :=\operatorname{ker}\left(l_{1}, l_{2}, \ldots, l_{s}\right) .
\end{aligned}
$$

By the compatibility $(2.7), l_{j} \phi_{1}=0$ for $j=2, \ldots, s$. Hence $\phi_{1} \in H^{\prime}$. Observe that $l_{1}$ is a linear mapping from $H$ to $H^{\prime}$ with $H^{\prime \prime}$ being its kernel. Hence

$$
\operatorname{dim}(H)=\operatorname{dim}\left(H^{\prime \prime}\right)+\operatorname{dim}\left(l_{1}(H)\right) .
$$

On the other hand, since $G$ possesses $s$-dimensional additivity, we have

$$
\operatorname{dim}(H)=\operatorname{dim}\left(H^{\prime \prime}\right)+\operatorname{dim}\left(H^{\prime}\right) .
$$


Comparing these two equations gives $l_{1}(H)=H^{\prime}$. Since $\phi_{1} \in H^{\prime}$, it follows that there exists an $f \in H$ such that $l_{1} f=\phi_{1}$. This $f$ also satisfies $l_{j} f=$ $0, j=2, \ldots, s$. Since $S$ is a direct sum of $M$ and $N, f$ has a unique decomposition $f=f_{1}+f_{2}$, where $f_{1} \in M$ and $f_{2} \in N$. But both $M$ and $N$ are invariant under $G$, hence the element $f_{1}$ satisfies $l_{1} f_{1}=\phi_{1}$ and $l_{j} f_{1}=0$, $j=2, \ldots, s$.

The general case will be proved by induction on $r$. Let $1 \leq r \leq s$ and assume that the theorem holds for $r-1$. When $r=1$, no compatibility assumption is made. Given $\phi_{1}, \ldots, \phi_{r} \in M$, we shall show that under the conditions of the theorem the system (2.6) has a solution $f$ in $M$. In the case $r=1$ we choose $f_{1}=0$. For $r>1$, by the induction hypothesis, we can find an $f_{1} \in M$ such that

$$
l_{i} f_{1}=\phi_{i}, \quad i=1, \ldots, r-1 .
$$

Let $g=l_{r} f_{1}$. Then $g \in M$ and

$$
l_{i} g=l_{i}\left(l_{r} f_{1}\right)=l_{r}\left(l_{i} f_{1}\right)=l_{r} \phi_{i}, \quad i=1, \ldots, r-1 .
$$

This, together with the compatibility conditions, implies

$$
l_{i}\left(\phi_{r}-g\right)=l_{i} \phi_{r}-l_{r} \phi_{i}=0, \quad i=1, \ldots, r-1 .
$$

Moreover, since $\left.l_{j}\right|_{M}(j=r+1, \ldots, s)$ are nilpotent, there exists a positive integer $m$ such that

$$
l_{j}^{m}\left(\phi_{r}-g\right)=0, \quad j=r+1, \ldots, s .
$$

Consider the following system of operator equations for $h$ :

$$
\begin{aligned}
l_{i} h & =0, \quad i=1, \ldots, r-1, \\
l_{r} h & =\phi_{r}-g \\
l_{j}^{m} h & =0, \quad j=r+1, \ldots, s .
\end{aligned}
$$

By (2.8) and (2.9), the compatibility conditions corresponding to the system (2.10) are satisfied. This is just the special case we discussed before. Therefore the system (2.10) has a solution $h$ in $M$. Let $f=f_{1}+h$. Then

$$
l_{i} f=l_{i} f_{1}+l_{i} h=\phi_{i}, \quad i=1, \ldots, r-1,
$$

and

$$
l_{r} f=l_{r} f_{1}+l_{r} h=g+\left(\phi_{r}-g\right)=\phi_{r} .
$$

This shows that $f$ is a solution to the system (2.6).

\section{SOLVABILITY OF DIFFERENTIAL AND DIFFERENCE EQUATIONS}

In this section, we prove that certain systems of differential and difference equations are solvable provided the conditions (1.2) are satisfied. This is done by applying the theorems established in $\S 2$ together with some basic concepts from algebraic geometry. We refer the reader to [L, Sh] for the necessary knowledge of algebraic geometry used in this section.

From now on $K$ is assumed to be an algebraically closed field of characteristic zero, and the ring of polynomials in $s$ indeterminates $X_{1}, \ldots, X_{s}$ over $K$ is denoted by $K\left[X_{1}, \ldots, X_{s}\right]=\Pi\left(K^{s}\right)$. 
For a given ideal $I$ of $K\left[X_{1}, \ldots, X_{s}\right]$ we denote by $V(I)$ the (affine) algebraic variety determined by $I$ :

$$
V(I):=\left\{a \in K^{s}: p(a)=0 \text { for all } p \in I\right\} .
$$

An algebraic variety $V$ is said to be reducible if it can be represented as a union of two algebraic varieties, both different from $V$. Otherwise, we call $V$ an irreducible algebraic variety. Given an algebraic variety $V$, we denote by $I(V)$ the ideal of all polynomials which vanish on $V$. The ring

$$
K[V]:=K\left[X_{1}, \ldots, X_{s}\right] / I(V)
$$

is called the coordinate ring of $V$. If $V$ is irreducible, then $K[V]$ is an integral domain. In this case the quotient field of $K[V]$ is called the field of rational functions on $V$, and is written $K(V)$.

(3.1) Definition. The dimension of an irreducible variety $V, \operatorname{dim}(V)$, is the transcendence degree of $K(V)$ over $K$. The dimension of a variety is the maximum of the dimensions of its irreducible components.

A single point has dimension 0 , and the dimension of any algebraic variety $V \subseteq K^{s}$ is at most $s$. Let $V \subseteq K^{s}$ be an algebraic variety. For a polynomial $f \in K\left[X_{1}, \ldots, X_{s}\right]$, we define the variety $V_{f}:=\{a \in V: f(a)=0\}$. We say that $f$ does not vanish on $V$ if $V_{f} \neq V$.

The following theorem is useful for proving some of the results in this section. The proof of this theorem can be found in [L, Chapter II, Theorem 11] and in [Sh, Chapter 1, §6, Theorem 4].

(3.2) Theorem (Dimension Theorem). Let $V$ be an irreducible variety of dimension $n \geq 1$ in $K^{s}$. If a polynomial $f \in K\left[X_{1}, \ldots, X_{s}\right]$ does not vanish on $V$ and $V_{f} \neq \varnothing$, then every irreducible component of $V_{f}$ has dimension $n-1$.

(3.3) Theorem. Suppose that every irreducible component of a variety $U$ in $K^{s}$ has the same dimension $n(n \geq 1)$. Let $\theta=\left(\theta_{1}, \ldots, \theta_{s}\right) \in U$. Then there exists an element $v=\left(v_{1}, \ldots, v_{s}\right) \in K^{s}$ such that

$$
F_{v}:=\left(X_{1}-\theta_{1}\right) v_{1}+\cdots+\left(X_{s}-\theta_{s}\right) v_{s} \in K\left[X_{1}, \ldots, X_{s}\right]
$$

does not vanish on any component of $U$. Consequently, every irreducible component of $U \cap V\left(F_{v}\right)$ has dimension $n-1$.

Proof. Let $U=U_{1} \cup \cdots \cup U_{m}$ be the decomposition of $U$ into irreducible components with $U_{i} \nsubseteq U_{j}$ for all $i \neq j$. Let

$$
L_{j}:=\left\{v=\left(v_{1}, \ldots, v_{s}\right) \in K^{s}: F_{v} \text { vanishes on all of } U_{j}\right\}, \quad j=1, \ldots, m .
$$

Clearly, $L_{j}$ is a linear subspace of $K^{s}$ for each $j$. The dimension of $L_{j}$ is at most $s-n$, for otherwise we would have $\operatorname{dim}\left(U_{j}\right)<n$. Since $n \geq 1$, the set $K^{s} \backslash \bigcup_{j=1}^{m} L_{j}$ is nonempty. For any $v \in K^{s} \backslash \bigcup_{j=1}^{m} L_{j}, F_{v}$ does not vanish on any of $U_{j}$ for each $j=1, \ldots, m$.

(3.4) Corollary. Let $U \subseteq K^{s}$ be an algebraic variety with all its irreducible components having the same dimension $n$. For any $\theta \in U$ there exist $n$ polynomials $p_{1}, \ldots, p_{n}$ of degree 1 such that they vanish at $\theta$ and such that $U \cap V\left(p_{1}, \ldots, p_{n}\right)$ is a finite set.

(3.5) Theorem. Let $p_{1}, \ldots, p_{r}(r \leq s)$ be polynomials on $K^{s}$. Assume that the variety $V\left(p_{1}, \ldots, p_{r}\right)$ is either empty or each of its irreducible components 
has dimension $s-r$. Then for given polynomials (resp. exponential polynomials) $\phi_{1}, \ldots, \phi_{r}$, the system of differential equations

$$
p_{j}(D) f=\phi_{j}, \quad j=1, \ldots, r,
$$

has a polynomial (resp. exponential polynomial) solution $f$ if and only if the following compatibility conditions hold:

$$
p_{j}(D) \phi_{k}=p_{k}(D) \phi_{j}, \quad 1 \leq j<k \leq r .
$$

Proof. Let $S$ be the linear space of all exponential polynomials on $K^{s}$ and $G:=G_{\Pi\left(K^{s}\right)}(D)$. It is clear that we only need to consider $\phi_{j} \in e_{\theta} \Pi\left(K^{s}\right)$ for some $\theta=\left(\theta_{1}, \ldots, \theta_{s}\right) \in K^{s}$ since $e_{\theta} \Pi\left(K^{s}\right)$ is $G$-invariant. Let $M=e_{\theta} \Pi\left(K^{s}\right)$. Given $\phi_{1}, \ldots, \phi_{r} \in M$, we consider the solvability for $f \in M$ of the system (3.6). If $p_{j}(\theta) \neq 0$ for some $j$, then $p_{j}(D)$ is invertible on $M$ (see Example (2.1)). Hence, the theorem follows from Theorem (2.4).

If $p_{j}(\theta)=0$ for all $j=1, \ldots, r$, then $\theta \in V\left(p_{1}, \ldots, p_{r}\right)$. By hypothesis, every irreducible component of $V\left(p_{1}, \ldots, p_{r}\right)$ has dimension $s-r$. Applying Corollary (3.4) to $V\left(p_{1}, \ldots, p_{r}\right)$, we see that there exist polynomials $p_{r+1}, \ldots, p_{s}$ of degree one such that they vanish at $\theta$ and the set

$$
V\left(p_{1}, \ldots, p_{r}, p_{r+1}, \ldots, p_{s}\right)
$$

is finite. In particular, all $p_{j}(D), j=1, \ldots, s$, are nilpotent on $M$. Thus, an application of Theorem (2.5) gives the desired result.

(3.7) Corollary. Let $p_{1}, \ldots, p_{s}$ be polynomials on $K^{s}$ such that $V\left(p_{1}, \ldots, p_{s}\right)$ is a finite set. The system of differential equations

$$
p_{j}(D) f=\phi_{j}, \quad j=1, \ldots, s,
$$

where the $\phi_{j}$ 's are exponential polynomials, has a solution in the space of exponential polynomials if and only if the following compatibility conditions hold:

$$
p_{j}(D) \phi_{k}=p_{k}(D) \phi_{j}, \quad 1 \leq j<k \leq s .
$$

Let $V \subseteq K^{s}$ be an algebraic variety. A subset $U \subseteq V$ is said to be closed if $U$ itself is an algebraic variety. A subset $O \subset V$ is said to be open if $V \backslash O$ is closed in $V$. Let $O$ be a nonempty open subset of an irreducible algebraic variety $V$. If a polynomial $f \in K\left[X_{1}, \ldots, X_{s}\right]$ vanishes on $O$, then it must vanish on $V$, for otherwise $V=(V \backslash O) \cup V_{f}$ gives a decomposition of $V$ with $V \backslash O \neq V$ and $V_{f} \neq V$. This contradicts the irreducibility of $V$. Thus, $I(O)=I(V)$. Hence, the coordinate ring $K[O]$ is the same as the coordinate ring $K[V]$. Consequently, the quotient field $K(O)$ of $K[O]$ is the same as $K(V)$. In particular, this shows that $\operatorname{dim}(O)=\operatorname{dim}(V)$ for any nonempty open subset $O$ of an irreducible variety $V$. Moreover, if $V$ is irreducible, then $K[O]=K[V]$ is an integral domain, hence $O$ is also irreducible.

Let $V$ be an algebraic variety and let $V=U_{1} \cup \cdots \cup U_{m}$ be a decomposition of $V$ into its irreducible components. For an open subset $O \subseteq V, O \cap U_{j}$ is open in $U_{j}$ for each $j=1, \ldots, m$. If $O \cap U_{j}$ is nonempty, then $O \cap U_{j}$ is an irreducible component of $O$. Thus, after discarding some possible empty sets,

$$
O=\left(O \cap U_{1}\right) \cup \cdots \cup\left(O \cap U_{m}\right)
$$

gives a decomposition of $O$ into its irreducible components. 
The results of Theorem (3.3) and its corollary can be extended to the case where $U$ is an open subset of an algebraic variety.

(3.8) Theorem. Let $O \subseteq K^{s}$ be an open subset of an algebraic variety with all its irreducible components having the same dimension $n$. Then for any $\theta \in O$ there exist $n$ polynomials $p_{1}, \ldots, p_{n}$ of degree 1 such that they vanish at $\theta$ and such that $O \cap V\left(p_{1}, \ldots, p_{n}\right)$ is a finite set.

Finally we establish the following theorem about difference equations.

(3.9) Theorem. Let $p_{1}, \ldots, p_{r}(r \leq s)$ be polynomials on $K^{s}$. Assume that the intersection of the variety $V\left(p_{1}, \ldots, p_{r}\right)$ with $(K \backslash\{0\})^{s}$ is either empty or each of its irreducible components has dimension $s-r$. Then for given polynomial sequences (resp. exponential polynomial sequences) $\phi_{1}, \ldots, \phi_{r}$, the system of difference equations

$$
p_{j}(\tau) f=\phi_{j}, \quad j=1, \ldots, r,
$$

has a polynomial sequence (resp. exponential polynomial sequence) solution $f$ if and only if the following compatibility conditions hold:

$$
p_{j}(\tau) \phi_{k}=p_{k}(\tau) \phi_{j}, \quad 1 \leq j<k \leq r .
$$

Proof. Let $S$ be the linear space of all exponential polynomial $K$-sequences on $\mathbb{Z}^{s}$. Without loss of generality, we may assume that

$$
\phi_{1}, \ldots, \phi_{r} \in \theta^{()} \Pi\left(K^{s}\right)=: M \text { for some } \theta=\left(\theta_{1}, \ldots, \theta_{s}\right) \in(K \backslash\{0\})^{s} .
$$

If $p_{j}(\theta) \neq 0$ for some $j$, then by Theorem (2.4), the system (3.10) has a solution in $M$ provided the compatibility conditions hold.

Suppose that $p_{j}(\theta)=0$ for all $j=1, \ldots, r$. Then $\theta \in V\left(p_{1}, \ldots, p_{r}\right)$, and hence each irreducible component of $V\left(p_{1}, \ldots, p_{r}\right) \cap(K \backslash\{0\})^{s}$ has dimension $s-r$ by the hypothesis. Again, we can find polynomials $p_{r+1}, \ldots, p_{s}$ of degree 1 such that they vanish at $\theta$ and the set

$$
V\left(p_{1}, \ldots, p_{r}, p_{r+1}, \ldots, p_{s}\right) \cap(K \backslash\{0\})^{s}
$$

is finite. Thus, an application of Theorem (2.5) gives the desired result.

\section{REFERENCES}

[DM $]$ W. Dahmen and C. A. Micchelli, On multivariate E-splines, Adv. Math. 76 (1989), 33-93.

$\left[\mathrm{DM}_{2}\right]$ - Local dimension of piecewise polynomial spaces, syzygies, and solutions of systems of partial differential equations, Math. Nachr. 148 (1990), 117-136.

[E] L. Ehrenpreis, Fourier analysis in several complex variables, Tracts in Math., vol. 17, WileyInterscience, New York, 1970.

[JRS] R. Q. Jia, S. D. Riemenschneider, and Z. Shen, Dimensions of kernels of linear operators, Amer. J. Math. 114 (1992), 157-184.

[L] S. Lang, Introduction to algebraic geometry, Interscience, New York, 1958.

[RJS] S. D. Riemenschneider, R. Q. Jia, and Z. Shen, Multivariate splines and dimensions of kernels of linear operators, Multivariate Approximation and Interpolation (W. Haussmann and K. Jetter, eds.), Birkhäuser Verlag, Basel, 1990, pp. 261-274. 
[Sh] I. R. Shafarevich, Basic algebraic geometry, Springer-Verlag, New York. 1974.

[S] Z. Shen, Dimension of certain kernel spaces of linear operators, Proc. Amer. Math. Soc. 112 (1989), 381-390.

Department of Mathematics, University of Alberta, Edmonton, alberta, Canada T6G 2G1

E-mail address, R.-Q. Jia: jiaexihu.math.ualberta.ca

E-mail address, S. Riemenschneider: shermeapprox.math.ualberta.ca

Center for the Mathematical Sciences, University of Wisconsin, Madison, Wisconsin 537305

Current address, Z. Shen: Department of Mathematics, National University of Singapore, 10 Kent Ridge Crescent, Singapore 0511 